\title{
Estudio comparativo de ansiedad entre muestras de estudiantes dominicanos y españoles
}

\section{Comparative study of anxiety in Spanish and Dominican students}

\author{
Zoilo Emilio García-Batista ${ }^{1}$, Antonio Cano-Vindel ${ }^{2}$ y Solmary Xiomara Herrera-Martínez ${ }^{3}$ \\ ${ }^{1}$ Pontificia Universidad Católica Madre y Maestra, República Dominicana. \\ ${ }^{2}$ Universidad Complutense de Madrid, España. \\ ${ }^{3}$ Universidad Arturo Michelena, Venezuela.
}

Disponible online 31 de diciembre de 2014

\begin{abstract}
El objetivo de la investigación fue comparar las manifestaciones de ansiedad entre estudiantes de medicina dominicanos y españoles. La muestra total estuvo conformada por 160 estudiantes de ambos países, 40 mujeres y 40 hombres en cada uno de los grupos, todos con una edad de 18 años, seleccionados de manera no probabilística de tipo intencional y teniendo como criterio de inclusión no haber sido diagnosticados por algún trastorno de ansiedad. Como instrumento de medición se aplicó el Inventario de Situaciones y Respuestas de Ansiedad-ISRA. Los datos fueron analizados mediante un ANOVA de dos factores (sexo y nacionalidad) el cual permitió hallar como resultado diferencias estadísticamente significativas por sexo para el sistema de respuesta cognitivo, fisiológico y nivel general de ansiedad, así como en áreas situacionales de ansiedad de evaluación y ansiedad en situaciones cotidianas. No se hallaron diferencias significativas por nacionalidad entre ambos grupos.

Palabras clave: Ansiedad; ISRA; Dominicanos; Españoles; Comparativo.

The aim of this study was to compare the manifestations of anxiety in Dominican and Spanish medical students. The total sample consisted of 160 students of both nationalities ( 80 students per group divided into 40 men and 40 women per group). All participants were more than 18 years old and were selected using a non-probabilistic intentional sampling method. The inclusion criterion was not having been previously diagnosed with an anxiety disorder. The assessment instrument used was the Inventory of Situations and Responses of Anxiety (ISRA). Data were analysed using ANOVA to determine statistically significant differences by gender and nationality. Differences by gender were found in cognitive and physiological responses, overall level of anxiety, and situational areas of anxiety assessment and anxiety in everyday situations. No significant differences were found by nationality.
\end{abstract}

Key words: Anxiety; ISRA; Dominicans; Comparative.

Correspondencia: Zoilo Emilio García Batista. Calle Nuestra Señora de Valvanera Edif. 123, piso 1D, 28047- Madrid, España. Teléfono: 809-5801962, extensión 4382, e-mail: zoiloegarcia@gmail.com. E-mails de los co-autores: Antonio Cano Vindel: canovindel@psi.ucm.es, Solmary Xiomara Herrera-Martínez: sohema55@hotmail.com 
La ansiedad es una reacción emocional implicada en los procesos de adaptación ante eventos aversivos o peligros anticipados. Existe un nivel de ansiedad que se considera normal e incluso útil, se le denomina umbral emocional y permite mejorar el rendimiento y la actividad. Sin embargo, cuando la ansiedad rebasa ciertos límites, aparece un deterioro de la actividad cotidiana (Celis et al., 2001).

El estudio de la ansiedad ha permeado la investigación del comportamiento humano en diferentes escenarios debido al papel importante que tiene al matizar las interacciones cotidianas de los individuos que tienden a preocuparse o a poner demasiada atención a situaciones negativas potencialmente peligrosas (Beck, Emery \& Greenberg, 1985, citados en Hernández-Pozo, Coronado, Araujo \& Cerezo, 2008).

El comportamiento ansioso afecta muchos aspectos de la vida cotidiana de una persona, como por ejemplo la eficiencia para resolver problemas, las interacciones con otras personas, la manera como se describen a sí mismos, su capacidad de relajación, su calidad de vida y, por ende, su salud (Cano \& Miguel, 2001).

La tendencia a la ansiedad tiene efectos que inciden en otros procesos psicológicos de forma negativa y que han inducido a una intensa investigación por parte de los especialistas, quienes la consideran un proceso intrincado y complejo que matiza al análisis cognitivo, ya que implica una interacción de esta disposición emocional con otros procesos psicológicos como la vigilia, la atención, la percepción, el razonamiento y la memoria. La ansiedad como una disposición, en su forma más pura, es un sentimiento difuso, sin objetivo, una sensación desagradable y persistente, que por lo general hace difícil para la persona que la experimenta, identificar su inicio y su fin (Hernández-Pozo, Coronado, Araujo \& Cerezo, 2008).

La ansiedad es uno de las patologías más frecuente a nivel mundial. Para la Asociación Americana de Psiquiatría (APA, 1994), por ejemplo, la prevalencia anual y de vida se ubican alrededor del 3 y del 5\%, respectivamente. En el Estudio Epidemiológico Europeo de los Trastornos Mentales (2004, citado en Alonso et al. 2004), conducido sobre una muestra de 21.000 personas, se registró una prevalencia de vida del 2,8\%. De acuerdo con la Encuesta Mundial de Salud Mental, desarrollada por la Organización Mundial de la Salud, la prevalencia anual de la ansiedad en Latinoamérica se ubicaría alrededor del $3,6 \%$, mientras que la de vida sería aproximadamente del 5,9\% (Rodríguez \& Vetere, 2010).

Arenas y Puigcerver (2009) señalan que los trastornos de ansiedad se encuentran dentro de las enfermedades más comunes de la actualidad, aclarando que son más frecuentes en el sexo femenino frente al masculino. Dentro de este tipo de patologías se encuentran el Trastorno de Ansiedad Generalizada (TAG), con una la tasa prevalencia-vida es del $4 \%$ en los hombres, frente al 7\% en las mujeres; Trastorno Obsesivo Compulsivo (TOC) afectando del 1 al 3\% de la población, Fobias, con una prevalencia-vida del $5 \%$ en los hombres y del
$11 \%$ en las mujeres; las fobias a situaciones como volar en avión, temor a los sitios cerrados o la altura, con una prevalencia-vida del $9 \%$ en hombres y un $12 \%$ en mujeres; y la fobia social, que se presenta en un $11 \%$ de los hombres frente al $16 \%$ de las mujeres. Y, el último tipo de patología, es el Trastorno por Estrés Postraumático (PTSD) con una prevalencia-vida del $10 \%$ en las mujeres frente al 5\% de los hombres.

Se destacan múltiples variables por las cuales puede surgir esta diferencia entre mujeres y hombres, tales como los factores psicosociales y socioculturales, que devienen en otros como el rol sexual, la pobreza y el nivel educativo. Además, en cada una de ellas se señalan diferencias de cada sexo, como lo son los efectos de los mismos trastornos previos, la predisposición genética, las hormonas sexuales, la reactividad endocrina frente al estrés, los sistemas de neurotransmisión y los determinantes neuropsicológicos, llegando a la conclusión que gran parte de las diferencias sexuales de las enfermedades anteriormente señaladas tienen auge psicobiológico. Todas estas variables pueden llevar a condicionar síntomas y el tratamiento que debe efectuarse para combatir la ansiedad en cada sexo (Sáez et al., 2013).

De acuerdo a investigaciones internacionales se ha encontrado que las niñas y las adolescentes presentan niveles significativamente más elevados de ansiedad escolar que los niños y los adolescentes (Birmaher et al., 1997; Burnham \& Gullone, 1997; Essau, Muris \& Ederer, 2002; Freudenthaler, Spinath \& Neubauer, 2008; Masi et al., 2000; Méndez, García- Fernández \& Olivares, 1996; Muris, 2002; Puskar, Sereika \& Haller, 2003; Steinhausen, Müller \& Winkler, 2008; Tejero, 2006). Estos estudios han argumentado que el predominio de ansiedad escolar en las mujeres podría ser explicado, entre otros factores, porque éstas presentan mayor libertad para demostrar sus emociones, debido a la educación recibida y a los estereotipos sociales (Méndez, 2005). Así, la sociedad les permite ante cualquier situación preocuparse, quejarse, llorar y sentirse vulnerables o frágiles ya que estas reacciones se consideran características socialmente adecuadas en el sexo femenino. Por el contrario, existe cierta permisividad social en cuanto a la manifestación de comportamientos agresivos y competitivos por parte de los varones (Méndez, Inglés \& Hidalgo, 2002).

Cabe mencionar que la ansiedad se puede manifestar de tres formas diferentes: a través de síntomas fisiológicos, cognitivos y conductuales. Éstos hacen referencia a la teoría del triple sistema de respuesta propuesta por Lang (1968). Los tres niveles distintos, pueden influirse unos en otros, es decir, los síntomas cognitivos pueden exacerbar los síntomas fisiológicos y éstos a su vez disparar los síntomas conductuales (Celis, Bustamante, Cabrera, Cabrera, Alarcón \& Monge, 2001).

Para medir el triple sistema de respuesta de ansiedad la comunidad científica ha desarrollado diferentes instrumentos fiables y válidos, uno de los más importantes es el ISRA (Miguel-Tobal y Cano-Vindel, 2002), un inventario en formato Situación-Respuesta, que permite evaluar la frecuencia con que 
se manifiestan una serie de respuestas cognitivas (pensamientos y sentimientos de preocupación, miedo, inseguridad, etc.), fisiológicas (diversos índices de activación del S.N.A. y S.N.S.) y motoras (varios índices de agitación motora) de ansiedad ante distintas situaciones (de evaluación, interpersonales, fóbicas y de la vida cotidiana); fundamentado conceptualmente en la teoría interactiva y la teoría conductual. La teoría interactiva surge como una reconciliación entre la teoría del rasgo y la teoría situacionista delineada por Endler (Endler \& Parker, 1992), quien propuso tres elementos básicos: primero, la conducta humana es función de continuos procesos multidireccionales entre persona-situación; segundo, los factores de tipo emocional, motivacional y cognitivos son los principales determinantes de la conducta de la persona; tercero, el significado psicológico que tiene la situación para la persona es igualmente determinante de la conducta (Cano \& Miguel, 1997; Endler \& Parker, 1992). Por tanto, los aspectos situacionales explican y predicen la conducta, siendo el individuo un agente activo e intencional en la interacción (Cano, Miguel, González \& Iruarrizaga, 1994; Endler \& Okada, 1975).

Desde esta perspectiva, la ansiedad se configura a partir de dos elementos principales: la ansiedad situacional, la cual es un estado transitorio del organismo que ocurre en respuesta a estímulos y varía de momento a momento, y la ansiedad como rasgo latente, propensión o disposición a responder ante situaciones de estrés de una manera relativamente constante (Endler \& Okada, 1975), siendo así el estado de ansiedad una función de la interacción de una dimensión específica del rasgo de ansiedad y un peligro situacional congruente (Endler \& Parker, 1992). De este modo, cualquier reacción de ansiedad depende o es función de la interacción entre el rasgo de ansiedad del individuo, las características estresantes de la situación en la que tiene lugar la conducta y la forma como la persona perciba e intérprete dichas situaciones (Cano \& Miguel, 1997).

De forma complementaria, la teoría conductual se ha referido a la ansiedad como un constructo que reúne un conjunto de respuestas motoras, psicofisiológicas y cognitivas provocadas por determinadas variables antecedentes, como estímulos del medio y respuestas del propio sujeto (Méndez \& Maciá, 1994) configurando el triple sistema de respuesta. En este sistema, las respuestas motoras más frecuentes corresponden a voz temblorosa y tics; las respuestas psicofisiológicas, a cambios cardiovasculares y elevación de la presión sanguínea, y las respuestas cognitivas a la presencia de pensamientos e imágenes que evalúan de manera negativa las situaciones, anticipando respuestas desfavorables (Méndez \& Maciá, 1994).

En cuanto a la teoría interactiva, esta permite evaluar la ansiedad, ya que a la luz de sus planteamientos es posible diseñar inventarios situación-respuesta que permitan dar cuenta de la variabilidad en las situaciones, las respuestas y la interacción entre ambas (Cano \& Miguel, 1997). Sobre el particular, Endler y Okada (1975) señalan que a partir de esta teoría es posible realizar la evaluación global de las diferencias individuales, ob- servando la ansiedad desde la persona en un contexto determinado, de tal manera que no sólo puede hablarse del rasgo sino de otros factores como los estímulos y la manera como éstos son percibidos dependiendo del alto o bajo rasgo de ansiedad; por lo tanto no puede considerarse a la persona $v s$ la situación de manera separada ya que estas variables están en continua interacción.

Acorde con estas perspectivas teóricas, se puede considerar la ansiedad como una reacción o respuesta emocional que abarca múltiples manifestaciones y que determina una transacción recíproca entre el individuo y el ambiente. Esta conceptualización es la que subyace al ISRA.

Innumerables investigaciones han utilizado el ISRA para determinar sus propiedades psicométricas y como instrumento de diagnóstico y evaluación en las diferentes áreas de la psicología. Tal es el caso de Amorim (1997), quien al realizar la baremación para población brasileña, llevó a cabo un análisis factorial de las respuestas, con resultados similares a los reportados en la prueba original, ya que retiene tres factores que agrupan los tres sistemas de respuesta independientes y explican el 55.6\% de la varianza. El análisis factorial de las situaciones explica el 50,17\% de la varianza y agrupa los ítems en cuatro factores con cierta independencia entre sí. En cuanto a la fiabilidad, señala un coeficiente Alfa de Cronbach entre 0,92 y 0,99 , y una correlación test-retest con valores que oscilan entre 0,74 y 0,88 .

En cuanto a las investigaciones en el campo clínico y de la salud se encuentran las de Copete, Jurado y Gómez (2000), quienes utilizaron el ISRA para hallar diferencias en los niveles de ansiedad entre personas con asma bronquial y sin ninguna enfermedad. Para ello utilizaron una muestra de 60 personas de la ciudad de Cali-Colombia, con edades comprendidas entre los 18 y 40 años. Los resultados señalaron niveles de ansiedad significativamente superiores en las personas que padecen asma. Por su parte, Cano y Miguel (2001) utilizaron el ISRA para medir ansiedad en personas con dificultades a nivel biológico, tales como hipertensión, estrés y cáncer, así como conductas de tabaquismo. Como conclusión señalan que las reacciones de ansiedad y los estados emocionales inciden en el deterioro de la salud.

Otra investigación fue la realizada por Moreno y Martín (2009) quienes aplicaron el ISRA con el objetivo de observar alteraciones emocionales relacionadas con la infertilidad y los tratamientos de técnicas de reproducción asistida (TRA), para lo cual evaluaron un grupo de personas infértiles y un grupo control de personas fértiles, encontrando que la población infértil presenta más altos grados de ansiedad motora antes de iniciar los TRA; también encontraron mayores niveles de ansiedad fisiológica y motora en las personas mayores de 30 años, con bajos niveles de escolaridad.

En el ámbito educativo Losiak (2005) evaluó con el ISRA los niveles de ansiedad de los estudiantes, tomando para ello una muestra de 258 sujetos y concluyendo que la ansiedad es 
un fenómeno cuyos altos niveles pueden presentarse en grupos no clínicos y ante situaciones relacionadas predominantemente con la vida diaria y particulares como montar en avión.

Por último, Furlan (2006) empleó este inventario en conjunto con otros instrumentos para evaluar la ansiedad y observar la influencia de dicho constructo en el rendimiento académico, señalando que el sistema de respuesta cognitivo es el que principalmente interfiere en las tareas que el estudiante debe realizar cuando se está preparando para un examen, tales como la codificación, la organización, el almacenamiento y la recuperación de la información.

En la actualidad, la valoración de los factores que intervienen en la génesis de la ansiedad y de sus manifestaciones constituye el objetivo de gran número de trabajos que pretenden delimitar su grado de influencia, buscando precisar las características de las manifestaciones de la ansiedad desde el punto de vista analítico, lo que permite profundizar en su conocimiento. El predominio de la ansiedad escolar en las mujeres y en la adolescencia temprana parece estar razonablemente demostrado en la actualidad. Sin embargo, en comparación con otros trastornos de ansiedad (Miguel-Tobal \& Cano-Vindel, 1995), la mayoría de estudios previos no han analizado las diferencias según sexo y nacionalidad teniendo en cuenta los factores situacionales y los sistemas de respuesta (cognitivo, psicofisiológico y motor) que configuran el constructo de ansiedad. El análisis de las diferencias según sexo y nacionalidad en cada una de las situaciones y sistemas de respuesta permitirá obtener un conocimiento más exhaustivo de este constructo de cara, por un lado, al posible establecimiento de datos normativos diferenciados según sexo y nacionalidad, y, por otro lado, la multidimensionalidad de la ansiedad nos permitiría establecer patrones de respuesta en las diferentes situaciones ansiógenas en función del sexo y la nacionalidad del sujeto. De este modo, al evaluar al sujeto ansioso, habrá que determinar qué manifestación predomina o cuál es su patrón de respuesta, tanto con fines descriptivos como comparativos.

Por ende, el propósito de este estudio ha sido comparar el rasgo de ansiedad, las manifestaciones en los tres sistemas de respuesta y en los cuatro factores situacionales (ansiedad de evaluación, interpersonal, situaciones fóbicas y situaciones de la vida cotidiana) obtenidos con el Inventario de Situaciones y Respuestas de Ansiedad (ISRA; Miguel-Tobal \& Cano-Vindel, 1994) en mujeres y hombres dominicanos y españoles estudiantes de medicina.

De acuerdo a lo planteado se espera que: (a) las mujeres presenten puntuaciones significativamente más altas que los hombres en todos los factores situacionales y de respuesta de la ansiedad, (b) que exista diferencias significativas en todos los factores situacionales y de respuesta entre españoles y dominicanos dadas las diferencias culturales existentes entre ambos países, y (c) que el grupo de españoles presente niveles de ansiedad significativamente más altos en todos los factores situacionales y de respuesta medidos por el ISRA.
Método
Participantes
La muestra total de este estudio fue de 160 estudiantes: 80 estudiantes (40 mujeres y 40 hombres) de primer año de medicina de la Pontificia Universidad Católica Madre y Maestra (PUCMM) de República Dominicana y 80 estudiantes (40 mujeres y 40 hombres) de primer año de medicina de la Universidad Complutense de Madrid (UCM) España, todos de 18 años de edad. Para la selección de la muestra se tomó como criterio de inclusión que los participantes no padecieran o se les hubiera diagnosticado algún trastorno de ansiedad con la finalidad de que formaran un grupo sano de población no clínica. Así mismo fueron seleccionados de manera no probabilística haciendo uso de una estrategia de muestreo intencional.

\section{Instrumentos}

Inventario de Situaciones y Respuestas de Ansiedad (ISRA, Miguel- Tobal \& Cano-Vindel, 2002). El ISRA, consta de 224 ítems, de los cuales 22 muestran diferentes situaciones de la vida cotidiana. Así mismo, siete de esos son respuestas cognitivas como: pensamientos negativos de sí mismo, temores ante la evaluación negativa, dificultades para concentrarse, inseguridad y preocupación; siete son respuestas a nivel motor, como: realización de movimientos repetitivos, evitación, consumo de diferentes sustancias y hacer cosas sin una finalidad concreta. Diez son las respuestas a nivel fisiológico, como: molestias en el estómago, nauseas, mareo, dificultades respiratorias y dolor de cabeza. Los 224 ítems son combinaciones entre dichas respuestas y las 22 situaciones. Finalmente, se incluye una pregunta abierta (número 23 ) en la cual se le solicita a la persona mencionar una situación diferente a las contempladas en el cuadernillo, que le produzca de manera frecuente alguna de las respuestas cognitivas, motoras o fisiológicas. Para responder el inventario, la persona debe evaluar la frecuencia con la que se presentan las respuestas a nivel cognitivo, motor o fisiológico ante cada una de las 22 situaciones, en una escala que va desde 0 a 4 , donde 0 , es casi nunca; 1, pocas veces; 2 , unas veces sí, otras veces no; 3 , muchas veces y 4 , casi siempre. En cuanto a la aplicación, la prueba tiene una duración de 40 a 60 minutos aproximadamente, se puede realizar de manera individual o colectiva. Respecto a la calificación del triple sistema de respuesta, se suman las puntuaciones de cada ítem perteneciente a cada una de las tres escalas (cognitiva, motora y fisiológica).

El ISRA posee unas características psicométricas muy adecuadas; presenta un alto coeficiente de fiabilidad test-retest de 0,81 , con un intervalo de dos meses (Cano-Vindel \& Miguel-Tobal, 1999), calculado en una muestra de 101 sujetos españoles. La consistencia interna puede considerarse muy alta, con valores que oscilan entre 0,92 y 0,99 , para la versión española, alcanzándose valores muy elevados en otras versiones. Los estudios de validez convergente (Cano-Vindel $\&$ Miguel-Tobal, 1999) han mostrado correlaciones altas en- 
tre el total del ISRA y el Manifest Anxiety Scale de Taylor (1953), alcanzando un valor de 0,69 en una muestra de 383 sujetos.

La estructura factorial del ISRA se ha estudiado tanto en el caso de las respuestas como de las situaciones, arrojando en ambos casos estructuras acordes con los modelos teóricos en los que se basa este instrumento. El análisis factorial de las 24 respuestas de ansiedad del ISRA arroja tres factores que coinciden básicamente con los tres sistemas de respuesta de ansiedad (Lang, 1968). Así, Miguel-Tobal y Cano-Vindel (1999) con una muestra de 1.209 sujetos, empleado el método P.C.A. (componentes principales) con rotación oblicua (DQUART), obtuvieron una solución de tres factores que explicaba el $64,7 \%$ de la varianza.

\section{Procedimiento}

Para la realización de este estudio, se extendió una invitación de forma voluntaria y anónima a los estudiantes para contestar el Inventario de Situaciones y Respuestas de AnsiedadISRA (Miguel-Tobal \& Cano-Vindel, 2002). Luego de que los participantes brindaran su consentimiento informado se procedió en primera instancia a la explicación de forma clara y breve de los objetivos de la investigación y en un segundo momento a la administración del instrumento de medición. La aplicación de la prueba se realizó en grupos de 20 personas, con un tiempo aproximado de 50 minutos en las sesiones de aplicación.

\section{Análisis de datos}

El análisis de datos incluye un análisis descriptivo sobre la variable ansiedad medida con el ISRA, como lo son la media y desviación típica. Asimismo, para evaluar las posibles diferencias en ansiedad de distintos grupos (en función de la nacionalidad y el sexo), se aplicó un ANOVA de dos factores siendo las variables independientes el sexo y la nacionalidad. El procesamiento de los datos se llevó a cabo a través del paquete estadístico Statistical Package for the Social Sciences (SPSS) versión 17.

\section{Resultados}

En la Tabla 1 se muestran los análisis descriptivos, medias y desviaciones típicas separadas por sexo y nacionalidad para los sistemas de respuestas (cognitivo, fisiológico y motor) y nivel general de ansiedad (T). En la Tabla 2 se observan las medias y desviaciones típicas para los rasgos o situaciones específicas generadoras de ansiedad (FI: ansiedad de evaluación, FII: ansiedad interpersonal, FIII: ansiedad fóbica y FIV: ansiedad interpersonal) en españoles y dominicanos, mujeres y hombres. En ambas tablas se puede observar que la mayor diferencia se presenta en el sexo, siendo las mujeres las que obtuvieron mayores puntuaciones tanto en los sistemas de respuestas como en los rasgos o situaciones especificas de ansiedad.
Tabla 1

Medias y desviaciones típicas de los diferentes grupos de la muestra por sexo y nacionalidad en los tres sistemas de respuesta y el nivel general de ansiedad $(N=160)$.

\begin{tabular}{|c|c|c|c|c|c|}
\hline \multirow{3}{*}{$\begin{array}{l}\text { Sistema de } \\
\text { Respuesta }\end{array}$} & & \multicolumn{4}{|c|}{ Grupos } \\
\hline & & \multicolumn{2}{|c|}{$\begin{array}{c}\underset{N=80}{\text { Dominicanos }} \\
\qquad\end{array}$} & \multicolumn{2}{|c|}{$\begin{array}{c}\text { Españoles } \\
N=\mathbf{8 0}\end{array}$} \\
\hline & & $\begin{array}{c}\text { Hombres } \\
N=\mathbf{4 0}\end{array}$ & $\underset{N=40}{\text { Mujeres }}$ & $\begin{array}{c}\text { Hombres } \\
\quad N=\mathbf{4 0}\end{array}$ & $\begin{array}{c}\text { Mujeres } \\
\boldsymbol{N}=\mathbf{4 0}\end{array}$ \\
\hline \multirow[t]{2}{*}{ Cognitivo } & $M$ & 51,98 & 65,28 & 48,40 & 67,80 \\
\hline & $D T$ & 25,00 & 30,92 & 24,02 & 29,32 \\
\hline \multirow[t]{2}{*}{ Fisiológico } & $M$ & 33,00 & 39,53 & 30,59 & 42,51 \\
\hline & $D T$ & 23,67 & 24,75 & 19,56 & 24,18 \\
\hline \multirow[t]{2}{*}{ Motor } & $M$ & 31,05 & 38,73 & 35,55 & 40,20 \\
\hline & $D T$ & 22,18 & 25,64 & 27,84 & 23,57 \\
\hline \multirow{2}{*}{$\begin{array}{l}\text { Nivel General de } \\
\text { ansiedad }\end{array}$} & $M$ & 116,03 & 143,53 & 114,54 & 150,51 \\
\hline & $D T$ & 62,48 & 68,10 & 67,65 & 70,26 \\
\hline
\end{tabular}

\section{Tabla 2}

Medias y desviaciones típicas de los diferentes grupos de la muestra por sexo y nacionalidad en las cuatro áreas situacionales o rasgos específicos $(N=160)$.

\begin{tabular}{|c|c|c|c|c|c|}
\hline \multirow{3}{*}{$\begin{array}{c}\text { Áreas } \\
\text { situacionales o } \\
\text { rasgos específicos }\end{array}$} & & \multicolumn{4}{|c|}{ Grupos } \\
\hline & & \multicolumn{2}{|c|}{$\begin{array}{c}\text { Dominicanos } \\
N=80\end{array}$} & \multicolumn{2}{|c|}{$\begin{array}{c}\text { Españoles } \\
N=80\end{array}$} \\
\hline & & $\begin{array}{c}\begin{array}{c}\text { Hombres } \\
N=\mathbf{4 0}\end{array} \\
\end{array}$ & $\begin{array}{c}\text { Mujeres } \\
N=\mathbf{4 0}\end{array}$ & $\begin{array}{c}\text { Hombres } \\
\quad N=\mathbf{4 0}\end{array}$ & $\begin{array}{c}\text { Mujeres } \\
N=40\end{array}$ \\
\hline \multirow{2}{*}{ FI } & $M$ & 55,30 & 68,05 & 54,18 & 75,33 \\
\hline & $D T$ & 30,56 & 28,78 & 32,16 & 35,12 \\
\hline \multirow{2}{*}{ FII } & $M$ & 12,60 & 14,62 & 15,25 & 17,93 \\
\hline & $D T$ & 10,15 & 10,99 & 10,54 & 11,44 \\
\hline \multirow{2}{*}{ FIII } & $M$ & 25,15 & 32,17 & 23,23 & 25,45 \\
\hline & $D T$ & 19,54 & 23,33 & 15,70 & 17,58 \\
\hline \multirow{2}{*}{ FIV } & $M$ & 11,80 & 16,48 & 11,70 & 18,97 \\
\hline & $D T$ & 8,06 & 12,92 & 10,73 & 12,35 \\
\hline
\end{tabular}

Nota. FI: ansiedad de evaluación, FII: ansiedad interpersonal, FIII: ansiedad fóbica y FIV: ansiedad interpersonal.

\section{Diferencias de sexo y nacionalidad en el sistema de respues- ta $y$ factores situacionales}

El análisis de varianza sobre los tres sistemas de respuestas Cognitivo (C), Fisiológico (F) y Motor (M) y a nivel de rasgo general de ansiedad $(\mathrm{T})$ presentó diferencias significativas en cuanto al sexo con los siguientes valores: en el sistema cognitivo $\left(F_{3,888}=14,17, p<, 001\right)$, en el sistema fisiológico $\left(F_{3,888}\right.$ $=6,36, p=, 013)$ y para el total o rasgo general $\left(F_{3,888}=8,93\right.$, $p=, 003)$. No obstante, no hubo diferencia de ansiedad entre sexos en el sistema motor. Por otro lado, no se revelaron diferencias estadísticamente significativas entre las nacionalidades española y dominicana (Ver Tabla 3).

El análisis de varianza realizado en los cuatro rasgos específicos o áreas generadoras de ansiedad (FI, FII, FIII y FIV), mostró diferencias estadísticas en cuanto al sexo, en situaciones de evaluación $\left(F_{3,888}=11,40, p=, 001\right)$ y situaciones de la vida cotidiana $\left(F_{3,888}=11,43, p=, 01\right)$ no existiendo diferencias en las situaciones interpersonales ni fóbicas. Tampoco se presentaron diferencias significativas en función de la nacionalidad (Ver Tabla 4). 
Tabla 3

Análisis varianza según nacionalidad y sexo en los tres sistemas de respuestas y el nivel general de ansiedad.

\begin{tabular}{cccccc}
\hline $\begin{array}{c}\text { Variables } \\
\text { dependientes }\end{array}$ & $\begin{array}{c}\text { Fuentes de } \\
\text { Variación }\end{array}$ & $\begin{array}{c}\text { Suma de } \\
\text { Cuadrados }\end{array}$ & $\begin{array}{c}\text { Media } \\
\text { Cuadrática }\end{array}$ & $\boldsymbol{F}$ & $\boldsymbol{p}$ \\
\hline Cognitivo & Nacionalidad & 11,03 & 11,03 &, 01 &, 904 \\
& Sexo & 10692,90 & 10692,90 & 14,17 &, 001 \\
Fisiológico & Nacionalidad & 3,31 & 3,31 &, 01 &, 937 \\
& Sexo & 3404,03 & 3404,03 & 6,36 &, 013 \\
Motor & Nacionalidad & 3,31 & 3,31 &, 01 &, 937 \\
& Sexo & 1519,06 & 1519,06 & 2,45 &, 120 \\
Nivel General & Nacionalidad & 302,50 & 302,50 &, 07 &, 796 \\
de ansiedad & Sexo & 40290,76 & 40290,76 & 8,93 &, 003 \\
\hline
\end{tabular}

Tabla 4

Análisis varianza según nacionalidad y sexo en las cuatro áreas situacionales o rasgos específicos.

\begin{tabular}{cccccc}
\hline $\begin{array}{c}\text { Variables } \\
\text { dependientes }\end{array}$ & $\begin{array}{c}\text { Fuentes de } \\
\text { Variación }\end{array}$ & $\begin{array}{c}\text { Suma de } \\
\text { Cuadrados }\end{array}$ & $\begin{array}{c}\text { Media } \\
\text { Cuadrática }\end{array}$ & $\boldsymbol{F}$ & $\boldsymbol{p}$ \\
\hline FI & Nacionalidad & 378,225 & 378,225 &, 375 &, 541 \\
& Sexo & 11492,100 & 11492,100 & 11,407 &, 001 \\
FII & Nacionalidad & 354,025 & 354,025 & 3,096 &, 080 \\
& Sexo & 220,900 & 220,900 & 1,932 &, 167 \\
\multirow{2}{*}{ FIII } & Nacionalidad & 748,225 & 748,225 & 2,020 &, 157 \\
& Sexo & 855,625 & 855,625 & 2,310 &, 131 \\
\multirow{2}{*}{ FIV } & Nacionalidad & 57,600 & 57,600 &, 461 &, 498 \\
& Sexo & 1428,025 & 1428,025 & 11,434 &, 001 \\
\hline
\end{tabular}

Nota. FI: ansiedad de evaluación, FII: ansiedad interpersonal,

FIII: ansiedad fóbica y FIV: ansiedad interpersonal. $\mathrm{P}<0,05$

\section{Discusión}

Diversas investigaciones han demostrado que el ISRA es un instrumento fiable y válido para evaluar la ansiedad en los tres sistemas de respuestas - cognitivo, fisiológico y motor - de forma general, así como en los cuatro rasgos específicos o áreas situacionales de ansiedad -situaciones de evaluación, interpersonales, fóbicas y cotidianas, apoyando firmemente los modelos teóricos que lo fundamentan (Endler, 1981; Lang, 1968).

En este estudio no se han detectado diferencias de los niveles de ansiedad entre la nacionalidad española y dominicana. Por otro lado, sí se detectaron diferencias significativas a nivel estadístico en cuanto al sexo.El análisis de varianza realizado señaló que existen diferencias en el sistema de respuesta cognitivo, fisiológico y general según la variable de sexo, siendo el grupo de mujeres el que mostró niveles de ansiedad más altos que el grupo de hombres.

También se detectaron diferencias significativas en función del sexo para el grupo de mujeres en los factores situacionales de ansiedad de evaluación y ansiedad en situaciones cotidianas. Estos resultados se muestran acordes con lo hallado por autores que destacan que las mujeres muestran niveles de ansiedad más elevados que los hombres. Tal es el caso de lo expuesto por Zubeidat, Fernández, Sierra y Salinas (2008) en donde señalan que las mujeres muestran una probabilidad significativamente mayor que los hombres de sufrir ansiedad. En esta línea, otros investigadores ya han informado que la asociación con diferentes diagnósticos, entre ellos el de ansiedad social, es signi- ficativamente mayor en mujeres que en hombres (La Greca \& López, 1998).

La investigación en el ámbito de la ansiedad implica dificultades por tratarse de un trastorno complejo y multifacético, y la propia definición y determinación de sus características es tarea complicada. La realización de estudios sobre factores asociados a la ansiedad como sexo y nacionalidad en estudiantes universitarios implica varios riesgos relacionados con el tipo de muestra seleccionada y con la metodología desarrollada, por lo que pueden obtenerse resultados contradictorios. Una de las limitaciones de este estudio es el tipo de muestra utilizada. Al no tratarse de una muestra clínica, no se pueden generalizar los resultados más allá de la población general. Además, se evaluaron estudiantes, por lo que no sabemos si los resultados son extensibles a jóvenes no escolarizados. La muestra tampoco se seleccionó aleatoriamente, sino que fue obtenida intencionalmente y por centros educativos que aceptaron participar en el estudio. Este tipo de muestreo incidental no asegura la representatividad de los resultados.

El presente estudio se ha categorizado como ex post facto de tipo descriptivo transversal. Este tipo de estudios carece de garantías suficientes para establecer relaciones causales inequívocas que relacionen las variables consideradas, por lo que las asociaciones encontradas se pueden interpretar en ambas direcciones o con base en terceras variables. En estudios transversales es posible establecer asociaciones entre variables, como las que aquí se han analizado, siempre y cuando su interpretación sea cautelosa. En cualquier caso, por la naturaleza transversal del estudio no es posible interpretar dichas asociaciones en el sentido de que una de las variables supone un riesgo para el desarrollo de la otra. Esto exigiría la realización de un estudio longitudinal de carácter prospectivo.

\section{Referencias}

1. Alonso, J., Angermeyer, M.C., Bernert, S., Bruffaerts, R., Brugha, T.S., Bryson, H., de Girolamo, G., Graaf, R., Demyttenaere, K., Gasquet, I., Haro, J.M., Katz, S.J., Kessler, R.C., Kovess, V., Lépine, J.P., Ormel, J., Polidori, G., Russo, L.J., Vilagut, G., Almansa, J., Arbabzadeh-Bouchez, S., Autonell, J., Bernal, M., Buist-Bouwman, M. A., Codony, M., Domingo-Salvany, A., Ferrer, M., Joo, S.S., Martínez-Alonso, M., Matschinger, H., Mazzi, F., Morgan, Z., ., Palacín, C., Romera, B., Taub, N. \& Vollebergh, W.A. (2004). Prevalence of mental disorders in Europe: results from the European Study of the Epidemiology of Mental Disorders (ESEMeD) Project. Acta Psychiatrica Scandinavica, 109, 21-27. http://dx.doi.org/10.1111/j.16000047.2004.00325.x

2. American Psychiatric Association. (1994). Manual diagnóstico y estadístico de los trastornos mentales. (4a ed.).Madrid: Masson.

3. Amorim, C. (1997). Estudio Transcultural de la Ansiedad: Adaptación del ISRA a la Población Brasileña. (Tesis Doc- 
toral inédita). Universidad Complutense de Madrid. Madrid.

4. Amorim-Gaudencio, C., Cano-Vindel, A. \& Miguel-Tobal, J.J. (1996). Diferenças na manifestação de ansiedade entre a população geral e pacientes com transtornos de pele. Psiquiatría Biológica, 4, 101-107.

5. Amorim-Gaudencio, C., Roustan, G. \& Sirgo, A. (2004). Evaluation of anxiety in chronic dermatose. Differences between sexes. Interamerican Journal of Psychology, 38, 103-112.

6. Arcas-Guijarro, S., \& Cano-Vindel, A. (1999). Procesos cognitivos en el trastorno de ansiedad generalizada, según el paradigma del procesamiento de la información. Recuperado el 2 de febrero de 2010 de http://www.psiquiatria. com/psicologia/vol143num141/art 146.htm

7. Arenas, M.C \& Puigcerver, A (2009). Diferencias entre hombres y mujeres en los trastornos de ansiedad: una aproximación psico-biológica. Escritos de Psicología, 3, 2029.

8. Bouayed, J., Rammal, H. \& Soulimani, R. (2009). Oxidative stress and anxiety. Oxidative Medicine and Cellular Longevity, 2, 63-67. http://dx.doi.org/10.4161/oxim.2.2.7944

9. Cano, A. \& Miguel, J. (1997). Inventario de situaciones y respuestas de ansiedad ISRA manual. (4a. Ed.). Madrid: TEA Ediciones.

10. Cano, A. \& Miguel, J. (2001). Emociones y salud. Ansiedad y Estrés, 7, 111-121. Recuperado el 6 de marzo de 2011, de http://www.psiquiatria.com/articulos/ansiedad/8652/

11. Cano, A., Miguel, J., González, H. \& Iruarrizaga, I. (1994). El afrontamiento de la ansiedad en las drogodependencias. Anales de Psicología 10, 145-156. Recuperado el 14 de febrero de 2008, de http://www.um.es/analesps/v10/ v10 2/05-10 2.pdf

12. Cano-Vindel, A. (2002). Técnicas cognitivas en el control del estrés. In E. G. Fernández-Abascal \& M. P. Jiménez Sánchez (Eds.), Control del Estrés (pp. 247-271). Madrid: UNED Ediciones.

13. Cano-Vindel, A. (2003). Desarrollos actuales en el estudio del control emocional / New developments in the study of emotional control. Ansiedad y Estrés, 9, 203-229.

14. Cano-Vindel, A., \& Miguel-Tobal, J.J. (1999). Evaluación de la ansiedad desde un enfoque interactivo y multidimensional: El inventario de Situaciones y Respuestas de Ansiedad (ISRA). Psicología Contemporánea, 6, 14-21.

15. Celis, J., Bustamante, M., Cabrera, D., Cabrera, M., Alarcón, W. \& Monge, E. (2001). Ansiedad y estrés académico en estudiantes de Medicina humana del primer y sexto año. Anales de la Facultad de Medicina, 62, 25-30.

16. Cohen, J. (1988). Statistical power analysis for the behavioral sciences. Hillsdale, NJ: Erlbaum.

17. Copete, A., Jurado, L. \& Gómez, I. (2000). Caracterización de la ansiedad en sujetos asmáticos de la ciudad de Cali a través del ISRA. Revista sonorense de Psicología, 14, 39-
49. Recuperado el 6 de marzo de 2011, de http://kunkaak. psicom.uson.mx/rsp/14-1-39-49.pdf

18. Endler, N. \& Okada, M. (1975). A multidimensional measure of trait anxiety: The S-R inventory of general trait anxiousness. Journal of Consulting and Clinical Psychology, 43, 319-329. http://dx.doi.org/10.1037/h0076643

19. Endler, N. \& Parker, J. (1992). Interactionism revisited: Reflections on the continuing crisis in the personality area. European Journal of personality, 6, 177-198. http://dx.doi. org/10.1002/per.2410060302

20. Espada, F., Torres, P., Cano-Vindel, A. \& Ochoa, E. (1994). Evaluación de trastornos de ansiedad: Diferenciación en los tres sistemas de respuesta y áreas situacionales. Clínica y Salud, 5, 209-217.

21. Espada, F. \& Cano-Vindel, A. (1999). Trastornos de ansiedad y trastorno depresivo: Diferencias en las manifestaciones de síntomas ansiosos y depresivos. Psicología Contemporánea, 6, 30-35.

22. Furlan, L. (2006). Ansiedad ante los exámenes. ¿Qué se evalúa y cómo?. Evaluar, 6, 32-51. Recuperado el 17 de abril de 2008, de http://revistas.unc.edu.ar/index.php/revaluar/article/view/533/473

23. García, J., Díaz, C. \& Aranda, J. (1993). Trastornos asociados al juego patológico. Anales de psicología, 9, 83-92.

24. Hair, J., Anderson, R., Tatham, R. \& Black, W. (2008). Análisis multivariante ( $5^{\mathrm{a}}$ ed.). Madrid: Prentice Hall Iberia.

25. Hernández-Pozo, M., Coronado, O., Araujo, V. \& Cerezo, S. (2008). Desempeño académico de universitarios en relación con ansiedad escolar y auto-evaluación. Acta Colombiana de Psicología. 11, 13-23.

26. Kessler, R. \& Ustun, T. (2004). The World Mental Health (WMH) Survey Initiative version of the World Health Organization (WHO) Composite International Diagnostic Interview (CIDI). International Journal of Methods in Psychiatric Research, 13, 93-121. http://dx.doi.org/10.1002/ $\underline{\text { mpr. } 168}$

27. La Greca, A. \& López, N. (1998). Social anxiety among adolescents: Linkages with peer relations and friendships. J Abnorm Child Psychol, 26, 83-94. http://dx.doi. org/10.1023/A:1022684520514

28. Lancha, C. \& Carrasco, M. (2003). Intervención en ansiedad a los exámenes, obsesiones y compulsiones contenidas en un trastorno obsesivo compulsivo de la personalidad. Acción Psicológica, 2, 173-190. http://dx.doi.org/10.5944/ ap.2.2.530

29. Lang, P. (1968). Fear reduction and fear behaviour: Problems in treating a construct. En Shilen J.M. (Ed): Research in pschotherapy vol.III. Washington: American Psichological Association.

30. Losiak, W. (2005). Shapes of Anxiety. Analysis of Anxiety Profiles Measured with S-R Questionnaire. Ansiedad y Estrés, 11, 157-162.

31. Martínez, K. \& Rodríguez, O. (2013). Análisis exploratorio 
de la validez del inventario de situaciones y respuestas de ansiedad (ISRA) adaptado. Acta Colombiana de Psicología, 16, 57-66.

32. McLean, C. \& Anderson, E. (2009). Brave men and timid women? A review of the gender differences in fear and anxiety. Clinical Psychology Review, 29, 96-505. http:// dx.doi.org/10.1016/j.cpr.2009.05.003

33. Méndez, F. \& Maciá, D. (1994). Evaluación de los problemas de ansiedad. En R. Fernández (Ed.), Evaluación Conductual Hoy: Un enfoque para el cambio en psicología clínica y de la salud. (pp. 426-460). Madrid: Pirámide.

34. Miguel-Tobal, J. \& Cano-Vindel, A. (1985). Evaluación de respuestas fisiológicas, cognitivas y motoras de ansiedad. Informes de Psicología, 4, 249-259.

35. Miguel-Tobal, J. \& Cano-Vindel, A. (1988). Inventario de situaciones y respuestas de ansiedad (ISRA): Manual ( $2^{\mathrm{a}}$. ed.). Madrid: TEA Ediciones.

36. Miguel-Tobal, J. \& Cano-Vindel, A. (1994). Inventario de situaciones y respuestas de ansiedad (ISRA): Manual $\left(3^{\mathrm{a}}\right.$. ed.). Madrid: TEA Ediciones.

37. Miguel-Tobal, J. \& Cano-Vindel, A. (1995). Perfiles diferenciales de los trastornos de ansiedad. Ansiedad y Estrés, 1, 37-60.

38. Miguel- Tobal, J. \& Cano-Vindel, A. (2002). Inventario de Situaciones y Respuestas de Ansiedad (ISRA). Madrid: TEA Ediciones.

39. Miguel-Tobal, J. \& Cano-Vindel, A. (2005). Estrés y Trabajo. In J. L. Arco Tirado (Ed.), Estrés y Trabajo: Cómo hacerlos compatibles (pp. 11-28). Sevilla: Instituto Andaluz de Administración Pública.

40. Míguez, M. \& Becoña, E. (2009). El consumo de tabaco en estudiantes de psicología a lo largo de 10 años (19962006). Psicothema, 21, 573-578. Recuperado el 8 de marzo de 2013, de http://www.psicothema.com/pdf/3673.pdf

41. Moreno, C. \& Martín, M. (2009). La ansiedad en parejas fértiles e infértiles. Ansiedad y Estrés, 15, 97-109.

42. Organización Mundial de la Salud. (2000). Comparación transnacional de la prevalencia de los trastornos mentales y los factores con ellos correlacionados. Bulletin of the World Health Organization, 78, 41.
43. Ortiz, J. (1992). Programa experimental del tratamiento cognitivo-conductual de la ansiedad (en una población de jóvenes delincuentes de entre 21 y 25 años). Análisis y Modificación de Conducta, 18, 519-537.

44. Rodríguez, R. \& Vetere, G. (2010). Ansiedad rasgo y creencias relacionadas con trastornos de ansiedad: una comparación entre el trastorno de ansiedad generalizada y otros cuadros de ansiedad. Perspectivas en Psicología, 7, 8-15.

45. Sáez, P., Araos, C., Escalona, C., Eluti, S., Del Rio, A., Martínez, J., Piffaut, A. \& Venegas, N. (2013). Comparación de los niveles de ansiedad y consumo de tabaco en jóvenes universitarios. Psicología Cientifica, 15, 8. Recuperado el 14 de abril de 2009, de http://www.psicologiacientifica. com/niveles-de-ansiedad-consumo-tabaco-jovenes-universitarios

46. Spielberger, C., Goursch, R. \& Lushene, R. (1994). Cuestionario de Ansiedad Estado- Rasgo: Manual (4a. Ed.). Madrid: TEA Ediciones.

47. Truyols, M., Palmer, A., Medinas, M., Pérez, J. \& Sesé, A. (2010). Emociones negativas en pacientes con lumbalgia crónica. Clínica y Salud, 21, 123-140. http://dx.doi. org/10.5093/cl2010v21n2a2

48. Wood, C., Cano-Vindel, A., Iruarrzaga, I. \& Dongil, E. (2009). Ansiedad y Tabaco. Intervención Psicosocial, 18, 213-231.

49. Zlomke, K. \& Hahn, K. (2010). Cognitive emotion regulation strategies: Gender differences and associations to worry. Personality and Individual Differences, 48, 408-413. http://dx.doi.org/10.1016/j.paid.2009.11.007

50. Zubeidat, I., Fernández, A., Sierra, J. \& Salinas, J. (2008). Evaluación de factores asociados a la ansiedad social y a otras psicopatologías en adolescentes. Salud Mental, 31, 189-196.

Fecha de recepción: 13 de octubre de 2013 Fecha de recepción de la versión modificada: 6 de octubre de 2014 Fecha de aceptación: 13 de octubre de 2014 